\title{
THEORY AND OBSERVATIONS OF COMETARY IONOSPHERES
}

\author{
T. E. Cravens \\ Space Physics Research Laboratory, Department of Atmospheric and Oceanic \\ Science, The University of Michigan, Ann Arbor, MI 48109, U.S.A.
}

\begin{abstract}
The physical and chemical processes responsible for cometary ionospheres are now beginning to be understood, due to comparisons between theoretical results and recently obtained in situ observations of the ionospheric plasma and magnetic field of comet Halley. The contact surface which separates outflowing cometary plasma from solar wind controlled cometary plasma can be explained in terms of a balance between the magnetic pressure gradient force and ion-neutral drag. An analytic expression for the magnetic field in the vicinity of the contact surface is given in this paper.
\end{abstract}

\section{INTRODUCTION}

Photoionization of cometary neutral atoms and molecules (e.g., $\mathrm{H}_{2} \mathrm{O}, \mathrm{OH}, \mathrm{O}, \mathrm{CO}_{2}$, etc.) by solar extreme ultraviolet radiation produces cometary ions which contaminate and mass-load the solar wind at large cometocentric distances. The plasma in the inner coma near the nucleus (i.e., ionospheric plasma) is purely cometary in origin and is strongly coupled to the neutral gas. Figure 1 is a schematic diagram illustrating the basic features of the plasma environment in the inner coma of a Halley -type comet (see the reviews by Mendis et. al. $/ 1 /$ and Ip and Axford $/ 2 /$ ). The location of the contact surface (abbreviated CS in this paper) is indicated. The plasma outside the CS is magnetized, and the ionospheric plasma inside is field-free. An inner shock might exist in order to divert the initially outwardly flowing plasma towards the tail, or as a consequence of the ionospheric electron temperature gradient $/ 1$.

This paper reviews our current understanding of cometary ionospheres. The next section contains a brief tutorial of the basic physical and chemical processes operating in cometary ionospheres. The third section is a review of some recent theoretical models of cometary ionospheres and the fourth section is a review of relevant in situ measurements of comets Halley and Giacobini-Zinner $(\mathrm{G} / \mathrm{Z})$ made by the GIOTTO and ICE (International Cometary Explorer) spacecraft, respectively. The fifth section is a general discussion of ionospheric plasma and in the sixth section the physical processes responsible for the formation of the contact surface (CS) are discussed.

\section{PHYSICAL AND CHEMICAL PROCESSES (TUTORIAL)}

The composition of the neutral inner coma, or atmosphere, of comet Halley near a heliocentric distance of $0.85 \mathrm{AU}$ was measured by the neutral mass spectrometer (NMS) onboard the GIOTTO spacecraft $/ 3 /$ and was shown to be about $80 \%$ or more $\mathrm{H}_{2} \mathrm{O}$ (by volume) with a total gas production rate of $\mathrm{Q}=6.9 \times 10^{29}$ molecules $/ \mathrm{s}$. The neutral outflow speed, $u_{n}$, was found to be about $0.9 \mathrm{~km} / \mathrm{s}$. Both the NMS and the ion mass spectrometer (IMS) $/ 4 /$ on GIOTTO have shown that $\mathrm{H}_{3} \mathrm{O}^{+}$is the major ion within a cometocentric distance, $\mathrm{r}$, of $\approx 1-2 \times 10^{4} \mathrm{~km}$, followed in abundance by $\mathrm{H}_{2} \mathrm{O}^{+}, \mathrm{OH}^{+}, S^{+}$, and $\mathrm{O}^{+}$, as well as many other species.

The neutral /dust dynamics in the inner coma is rather complicated but for values of $\mathrm{r}$ greater than $1 \mathrm{~km}$ or so the neutral outflow speed, $u_{n}$, is an approximately constant $1 \mathrm{~km} / \mathrm{s}$. The total neutral density, $n_{n}$, is then:

$$
n_{n}=Q /\left(4 \pi u_{n} r^{2}\right)
$$

These neutrals can be ionized by solar extreme ultraviolet (EUV) radiation, by photoelectrons, and possibly by energetic electrons $/ 5,6 \%$

$$
\text { hv (ore) }+\mathrm{H}_{2} \mathrm{O} \longrightarrow \mathrm{H}_{2} \mathrm{O}^{+}+\mathrm{e}
$$

The neutral density at large radial distances $\left(\approx 10^{6} \mathrm{~km}\right)$ is attenuated by these ionization processes, but at smaller radial distances equation (1) is reasonably accurate. As is well-known from theory $\Pi, 8 \%$, the $\mathrm{H}_{2} \mathrm{O}^{+}$ions rapidly react with $\mathrm{H}_{2} \mathrm{O}$ to produce $\mathrm{H}_{3} \mathrm{O}^{+}$:

$$
\mathrm{H}_{2} \mathrm{O}^{+}+\mathrm{H}_{2} \mathrm{O} \longrightarrow \mathrm{H}_{3} \mathrm{O}^{+}+\mathrm{OH} \quad \mathrm{k}_{3}=2 \times 10^{-9} \mathrm{~cm}^{3} \mathrm{~s}^{-1}
$$




\section{INNER COMETARY PLASMA ENVIRONMENT}

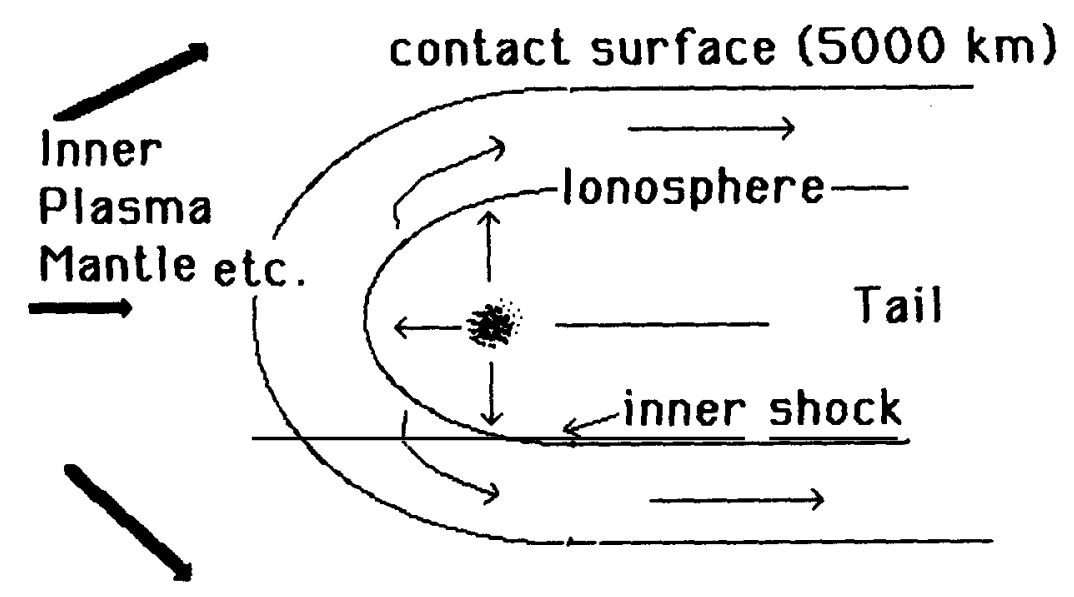

Fig. 1. Schematic of inner plasma environment of comet Halley.

The $\mathrm{H}_{3} \mathrm{O}^{+}$ions rapidly recombine dissociatively:

$$
\mathrm{H}_{3} \mathrm{O}^{+}+\mathrm{e} \longrightarrow \mathrm{H}_{2} \mathrm{O}+\mathrm{H} \quad \alpha=7 \times 10^{-7}\left(300 / \mathrm{T}_{\mathrm{e}}\right)^{1 / 2}
$$

where $T_{e}$ is the electron temperature. Similar chemical reactions apply to the other ion species.

The continuity equation for ion species $j$ with velocity, $u_{j}$, and number density, $n_{j}$, is

$$
\partial n_{j} / \partial t+\nabla \cdot\left(n_{j} u_{j}\right)=P_{j}-L_{j} n_{j}
$$

where $P_{j}$ and $L_{i} n_{j}$ are the chemical production and loss rates. $u_{j}$ in the inner ionosphere is radially outward with a magnitude $u_{j}$ apptoximately equal to $1 \mathrm{~km} / \mathrm{s} / 8 /$. An ion species is photochemically controlled if its chemical lifetime $\left(\tau_{c}=1 / L_{j}\right)$ is much less than its transport time $\left[\tau_{t r a n s}=r / u_{j}\right.$ where the relevant length scale is $\left.r\right]$. The photochemical expression for the density is just $n_{j}=P_{j} L_{j}$.

Now consider the $\mathrm{H}_{2} \mathrm{O}^{+}$ion. The production rate of this ion is $\mathrm{P}_{\mathrm{H}_{2} \mathrm{O}^{+}}=\mathrm{In}_{\mathrm{n}}$, where $\mathrm{I} \approx 10^{-6} \mathrm{~s}^{-1}$ is the total $\mathrm{H}_{2} \mathrm{O}$

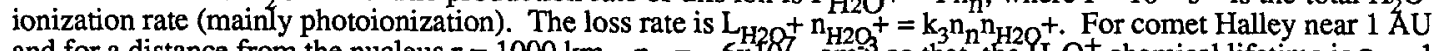
and for a distance from the nucleus $\mathrm{r}=1000 \mathrm{~km}, \mathrm{n}_{\mathrm{n}}=6 \times 18^{7} \mathrm{~cm}^{-3}$ so that the $\mathrm{H}_{2} \mathrm{O}^{+}$chemical lifetime is $\tau_{\mathrm{c}}=1$ $/ \mathrm{k}_{3} \mathrm{n}_{\mathrm{n}} \approx 10 \mathrm{~s}$. This value of $\tau_{\mathrm{c}}$ is much less than the transport time of about $1000 \mathrm{~s}$. In fact, the $\mathrm{H}_{2} \mathrm{O}^{+}$ion should be photochemically controlled everywhere within a distance of a few $x 10^{4} \mathrm{~km}$. Its density is:

$$
\mathrm{n}_{\mathrm{H} 20^{+}}=\left(\mathrm{I} \mathrm{n}_{\mathrm{n}}\right) /\left(\mathrm{k}_{3} \mathrm{n}_{\mathrm{n}}\right) \approx 500 \mathrm{~cm}^{-3}
$$

Notice that the density is independent of $r$ since the production and loss rates are both proportional to $n_{n}$.

$\mathrm{H}_{3} \mathrm{O}^{+}$is the major ion in the cometary ionosphere, and $\mathrm{n}_{3} \mathrm{O}^{+} \approx \mathrm{n}_{\mathrm{e}}$ where $\mathrm{n}_{\mathrm{e}}$ is the electron density. Assume initially that this species is photochemically controlled. Its production rate is almost equal to the $\mathrm{H}_{2} \mathrm{O}^{+}$production

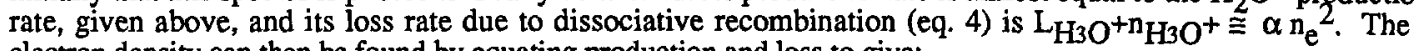
electron density can then be found by equating production and loss to give:

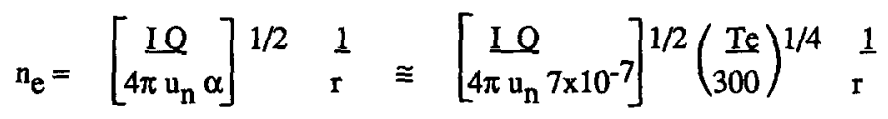

Using parameters given earlier from GIOTTO for comet Halley, this expresssion becomes

$$
n_{e}=10^{12}\left(T_{e} / 300\right)^{1 / 4}(1 / r)
$$

As has been well-known from theory $/ 1,2 /$, the photochemical electron density varies inversely with the distance from the nucleus. And the total ion current (proportional to $n_{\mathrm{e}}$ ) measured by the GIOTTO ion mass spectrometer $/ 4$ / varies as $1 / \mathrm{r}$ overall. 
It was assumed in deriving equation (7) that $\mathrm{H}_{3} \mathrm{O}^{+}$was photochemically controlled. But is this a good assumption? The recombination time is $\tau_{c}=1 / \alpha n_{e} \approx\left(10^{-5} r\right)$, and the transport time is $\tau_{\text {trans }}=r / u_{i} \approx\left(10^{-5} r\right)$. Both these time constants are comparable for any value of $r$, indicating that transport is also important. One can get a feeling for how transport affects the electron density profile by finding an expression for $\mathrm{n}_{\mathrm{e}}$ that neglects recombination altogether. The solution to the continuity equation (eq. 5) with zero loss rate and with the approximation that $u_{i}=1$ $\mathrm{km} / \mathrm{s}$ is:

$$
\mathrm{n}_{\mathrm{e}}=\left(\begin{array}{c}
\underline{\mathrm{L}} \\
4 \pi \mathrm{u}_{\mathrm{n}}
\end{array}\right)\left(\begin{array}{c}
1 \\
\mathrm{u}_{\mathrm{i}}
\end{array}\right) \quad \begin{aligned}
& \mathrm{r} \\
& \mathrm{r}
\end{aligned}
$$

which becomes the following for comet Halley:

$$
\mathrm{n}_{\mathrm{e}} \cong 6 \times 10^{12} / \mathrm{r}
$$

The radial dependence of $n_{e}$ is still $1 / r$. Gombosi et al. /9/ derived an analytic expression for $n_{e}$ which included both transport and recombination and this indicates that equation (7) is a much better approximation to $n_{e}$ than equation (8).

The electrons and ions are strongly coupled to the neutral gas in the inner coma where the neutral density is high and where the electron-neutral and ion-neutral energy exchange rates are large. All the temperatures are about the same in the inner coma: $T_{e} \cong T_{i} \cong T_{n}$. The electron and ion temperatures become significantly higher than the neutral temperature only at larger values of $\mathrm{r}$.

\section{REVIEW OF RECENT THEORETICAL MODELS}

Some recent theoretical models of cometary ionospheres are reviewed in this section; more comprehensive reviews can be found in Mendis et al. /1/ and Huebner et al. /10/. The Marconi and Mendis model (designated MM) $/ 1,11,12 /$ will be discussed first and then the Körösmezey et al. $/ 8 /$ model. Both these models are one-dimensional with radial outflow. The MM model treats the heavy neutrals, atomic hydrogen, the dust, and the thermal electrons as separate "fluids", but combines the ions with the heavy neutrals. Continuity, momentum, and energy equations for each fluid were solved; however, the electron velocity was assumed to be the same as the neutral / ion velocity. The parent molecule composition of the gas sublimating from the nucleus for this model can vary but for the results reviewed here was $85 \% \mathrm{H}_{2} \mathrm{O}, 10 \% \mathrm{CO}_{2}$, and $5 \% \mathrm{~N}_{2}$. Multiple scattering of the incoming radiation was also taken into account.

Figure 2 shows the calculated temperature profiles for a Halley-type comet. The neutral temperature initially decreases due to adiabatic cooling as the gas expands outward from the nucleus, but further out $T_{n}$ increases due to photochemical heating. The electron temperature is equal to $T_{n}$ for $r$ less than about $2000-\mathrm{km}$ but then increases rapidly for two reasons: (1) reduced electron-neutral cooling at large distances and, (2) heating from Coulomb collisions of the thermal electrons with the superthermal (energies $\mathrm{E}=10 \mathrm{eV}$ ) photoelectrons produced by reaction (2). Heat conduction significantly reduces the electron temperature by transporting heat away from larger cometocentric distances down to the collision-dominated inner coma where the electrons are more efficiently cooled.

Electron and ion density profiles from the MM model are shown in Figure 3. The densities are small for $\mathrm{r}<50-100$ $\mathrm{km}$ due to the opacity of this inner region for solar EUV radiation. The major ion is $\mathrm{H}_{3} \mathrm{O}^{+}$and its density varies approximately as the inverse of $r$, for the reasons given in section 2 (eq. (7)). The electron density increases at a cometocentric distance where the electron temperature increases as one would expect from equation (7). For $r>100$ $\mathrm{km}$, the $\mathrm{H}_{2} \mathrm{O}^{+}$density is almost constant, as discussed in the tutorial (eq. (6)).

The MM model has also been applied to comet $\mathrm{G} / \mathrm{Z} / 7 /$ which has a total gas production rate $\mathrm{Q}=2-3 \times 10^{28} \mathrm{~s}^{-1}$. The critical assumption for this application is that the ionospheric plasma be able to flow unimpeded down the tail. The calculated electron density and temperature at a cometocentric distance of about 7800-km (the ICE spacecraft crossed the tail axis at this distance) are $\approx 2000 \mathrm{~cm}^{-3}$ and $\approx 20,000 \mathrm{~K}$, respectively, and are in reasonably good agreement with the values measured in situ by the ICE spacecraft (shown in the next section).

Another model of the ionosphere of comet Halley has recently been developed by Korosmezey et al. /8/. In this model (designated $\mathrm{K}$ ), the coupled continuity, momentum, and energy equations for electrons and ions were solved using a numerical method that permits shocks. The ions are handled as a seperate species from the neutrals, with their own velocity and temperature. The $\mathrm{K}$ model only considers the ionospheric plasma and adopts all neutral atmosphere parameters from the work of Gombosi et al. /13/. The photoelectron flux as a function of electron energy and distance is calculated in detail and is used to determine the heating rate of the ambient thermal ionospheric electrons. This heating rate was used in the calculation of the electron and ion temperatures. Calculated photoelectron fluxes are displayed in Figure 4 for two values of $r$. The structure in these photoelectron spectra results from structure in the solar EUV spectra. 


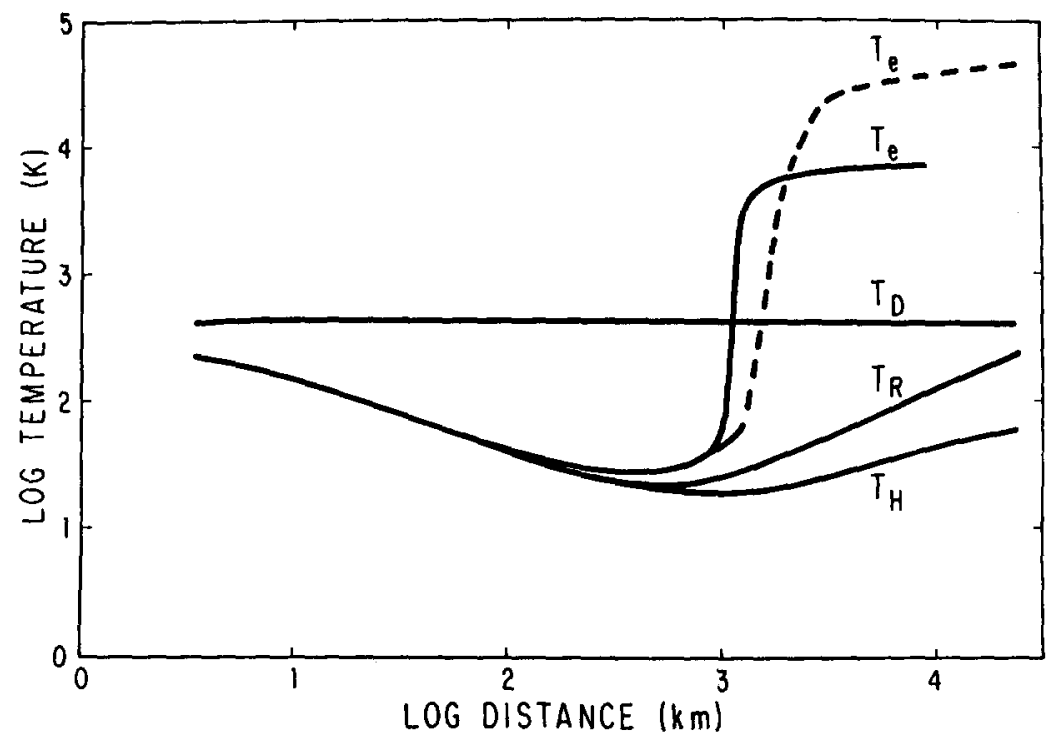

Fig. 2. Temperatures of electrons $\left(T_{e}\right)$ heavy neutrals $\left(T_{R}\right)$, hydrogen $\left(T_{H}\right)$, and the dust $\left(T_{D}\right)$ as functions of cometocentric distance, from the theoretical model of Marconi and Mendis (cf. /1/).

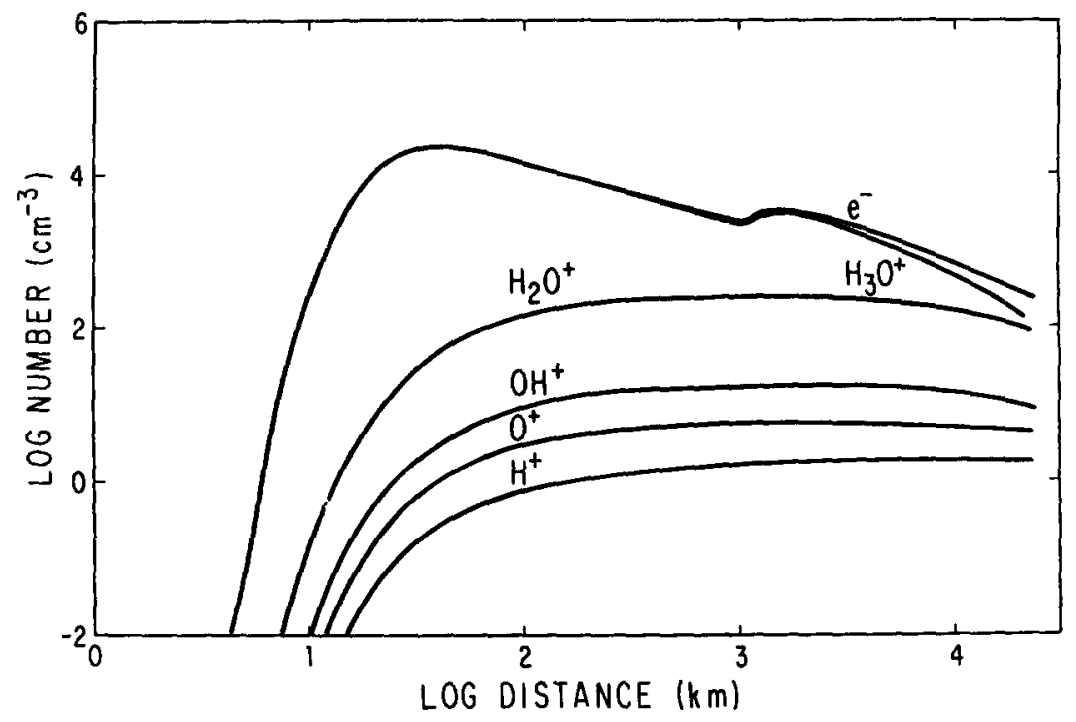

Fig. 3. Electron and ion densities from the Marconi and Mendis model.

The electron temperatures calculated in the $\mathrm{K}$ model (not shown here) are qualitatively similar to those calculated in the MM model but quantitatively are considerably lower than the MM temperatures at larger distances. The electron temperature calculated in the $\mathrm{K}$ model is only $\approx 1000 \mathrm{~K}$ at $10^{4} \mathrm{~km}$ if no external heat inputs are included, whereas in the $M M$ model $T_{e}$ is several thousand degrees. The electron heating efficiency due to photoelectrons is considerably smaller in the $\mathrm{K}$ model than in the $\mathrm{MM}$ model because most of the photoelectron energy is lost via collisions with neutrals rather than with the thermal electrons. However, a $M M$ type of $T_{e}$ profile is obtained by Körösmezey et al. if a external heat input into the electrons of $3 \times 10^{9} \mathrm{eV} / \mathrm{cm}^{2} / \mathrm{s}$ is applied at the upper boundary at $10^{4} \mathrm{~km}$. The ion temperature calculated at $10^{4} \mathrm{~km}$ is also about $1000 \mathrm{~K}$ and is larger than the neutral temperature. The ions are primarily heated by the exothermic energy associated with chemical reactions such as reaction (3). 


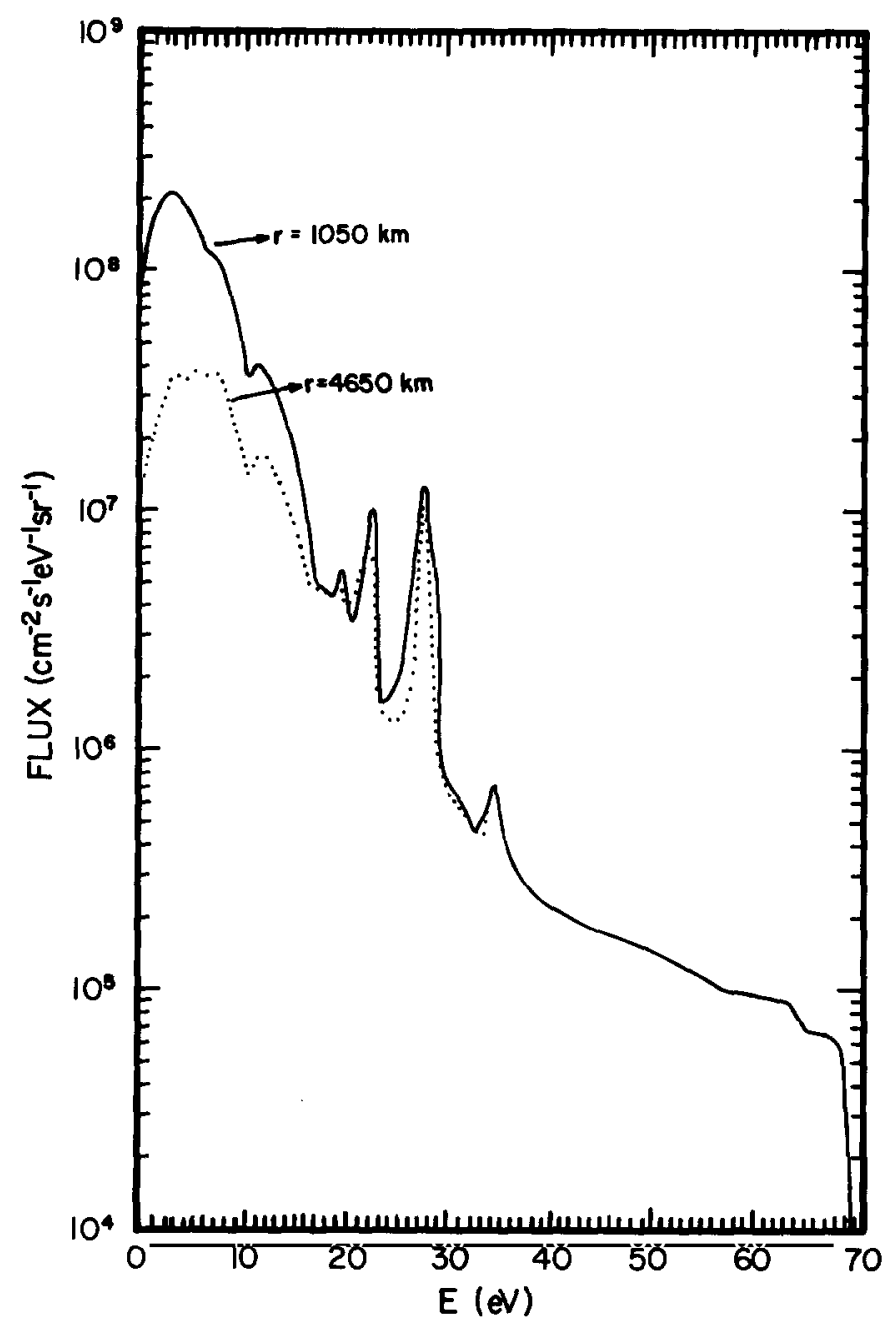

Fig. 4. Theoretical cometary photoelectron fluxes versus electron energy at two cometocentric distances, from the model of Körösmezey et al. $/ 8 \%$.

The plasma velocity is permitted to be different from the neutral velocity in the $\mathrm{K}$ model although the velocities are almost the same $\left(u_{i} \cong u_{n}\right)$ in the collision-dominated inner region (see Figure 5 ). The ion velocity begins to decrease relative to the neutrals near $\mathrm{r} \approx 1000 \mathrm{~km}$ because the increasing electron temperature results in an electron pressure gradient which produces an inwardly directed polarization electric field which decelerates the ions. This decrease in $\mathrm{u}_{\mathrm{i}}$ is smooth for the cases with only internal electron heating, but there is a sharp shock-like decrease of $\mathrm{u}_{\mathrm{i}}$ (an inner shock?) for the case with the external heat input. The electron density profile calculated in the $\mathrm{K}$ model is qualitatively very similar to the $M M$ profile and $n_{e}$ varies approximately as $1 / r$ with a slight enhancement where $\mathrm{T}_{\mathrm{e}}$ increases.

\section{IN SITU OBSERVATIONS OF COMETARY IONOSPHERES}

In situ experimental measurements of the ionospheres of comet Halley and comet Giacobini-Zinner are now available, for the first time. The ICE spacecraft observed a narrow plasmasheet of cold dense plasma (thickness $\approx$ $2000 \mathrm{~km}$ ) as it traversed the neutral sheet part of the magnetotail $/ 14,15 \%$. In the plasmasheet, $\mathrm{n}_{\mathrm{e}} \approx 10^{3} \mathrm{~cm}^{-3}$ and $\mathrm{T}_{\mathrm{f}}$ $\approx 10^{4} \mathrm{~K}$ (see Fig. 6). It seems reasonable to conclude that this is ionospheric plasma convected down the the taif $n 1$.

The GIOTTO spacecraft first entered a magnetic field-free cavity at a cometocentric distance of about $4500 \mathrm{~km}$ and exited this cavity at about the same distance after closest approach /16/ (see Figure 7). This cavity can be identified as the ionospheric region and the ion mass spectrometer (IMS) on GIOTTO made measurements throughout this region /4/. The composition from the IMS was alluded to earlier in this paper and the total ion counting rate (assumed to be proportional to $\mathrm{n}_{\mathrm{e}}$ ) is reproduced in Figure 8. The total ion counting rate varies approximately as the 


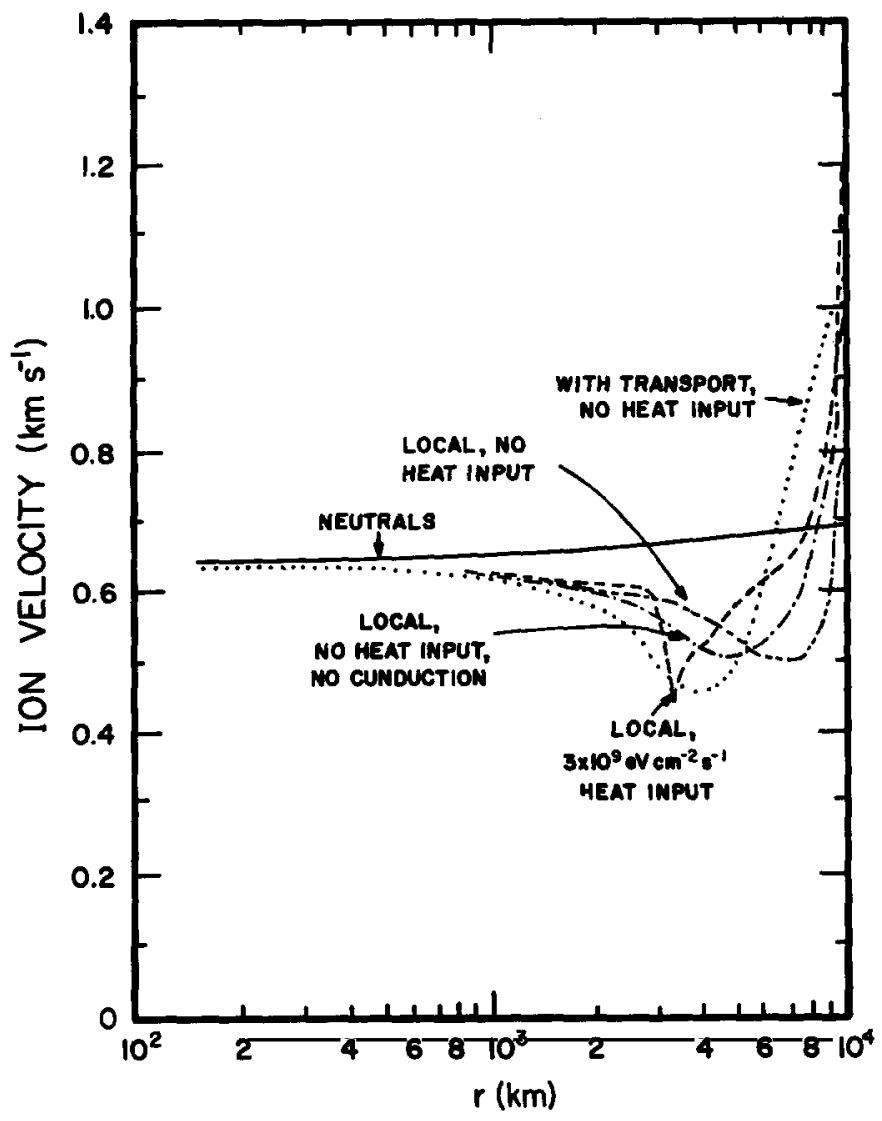

Fig. 5. Calculated velocities versus cometocentric distance from $/ 8 /$. Results for four different sets of conditions are shown (local or transport for photoelectrons, heat input or no input at the upper boundary, etc.).

inverse power of $\mathbf{r}$ for all values of $\mathbf{r}$ less than about $10^{4} \mathrm{~km}$. In fact, there is only a slight deviation from this $r$-dependence at the contact surface (CS), indicating the CS is not a Venus-type ionopause where the thermal plasma density and pressure decrease. Plasma flow speeds and ion temperatures measured by the IMS are shown in Figure 9. The ion flow speed decreases from about $1 \mathrm{~km} / \mathrm{s}$ inside the ionosphere to approximately zero in the vicinity of the $C S$, and $T_{i}$ varies from $\approx 400 \mathrm{~K}$ inside the ionosphere to about $\approx 2500 \mathrm{~K}$ near the $C S$.

\section{IS THE PLASMA OUTSIDE THE CONTACT SURFACE IONOSPHERIC?}

By what criteria is a plasma commonly called "ionospheric" by terrestrial or planetary standards ? The plasma should possess the following interrelated properties (by comparison with solar wind or magnetospheric plasma):

(1) Slow, (2) Dense, (3) Cold, (4) Interacts strongly with neutrals

The outwardly flowing plasma in the region below the CS certainly has these properties and has always been referred to as the cometary "ionosphere" (cf. /1). But what about the plasma in the region just outside the CS (4500 $\mathrm{km} \leq \mathrm{r} \leq 2 \times 10^{4} \mathrm{~km}$ ) ? The GIOTTO measurements (reviewed in the last section) indicate that:

(1) The flow speed is less than $1-2 \mathrm{~km} / \mathrm{s}$ (SLOW).

(2) The measured electron density just outside the CS is comparable to the density below the contact surface $\left(\mathrm{n}_{\mathrm{e}} \approx\right.$ $10^{3} \mathrm{~cm}^{-3}$ is expected from theory). The plasma is DENSE.

(3) The measured ion temperatures are less than $=10^{4} \mathrm{~K}$. The electron temperature in this region is expected to be less than $1-2 \times 10^{4} \mathrm{~K}$ from theory $/ 17,18 /$ and from ICE measurements in the tail of comet $\mathrm{G} / \mathrm{Z} / 15 /$. The plasma is COLD.

(4) The major ion in this region was observed $/ 4 /$ to be $\mathrm{H}_{3} \mathrm{O}^{+}$, which indicates that the reaction of $\mathrm{H}_{2} \mathrm{O}^{+}$with $\mathrm{H}_{2} \mathrm{O}$ proceeds rapidly. Interactions with NEUTRALS are important. 


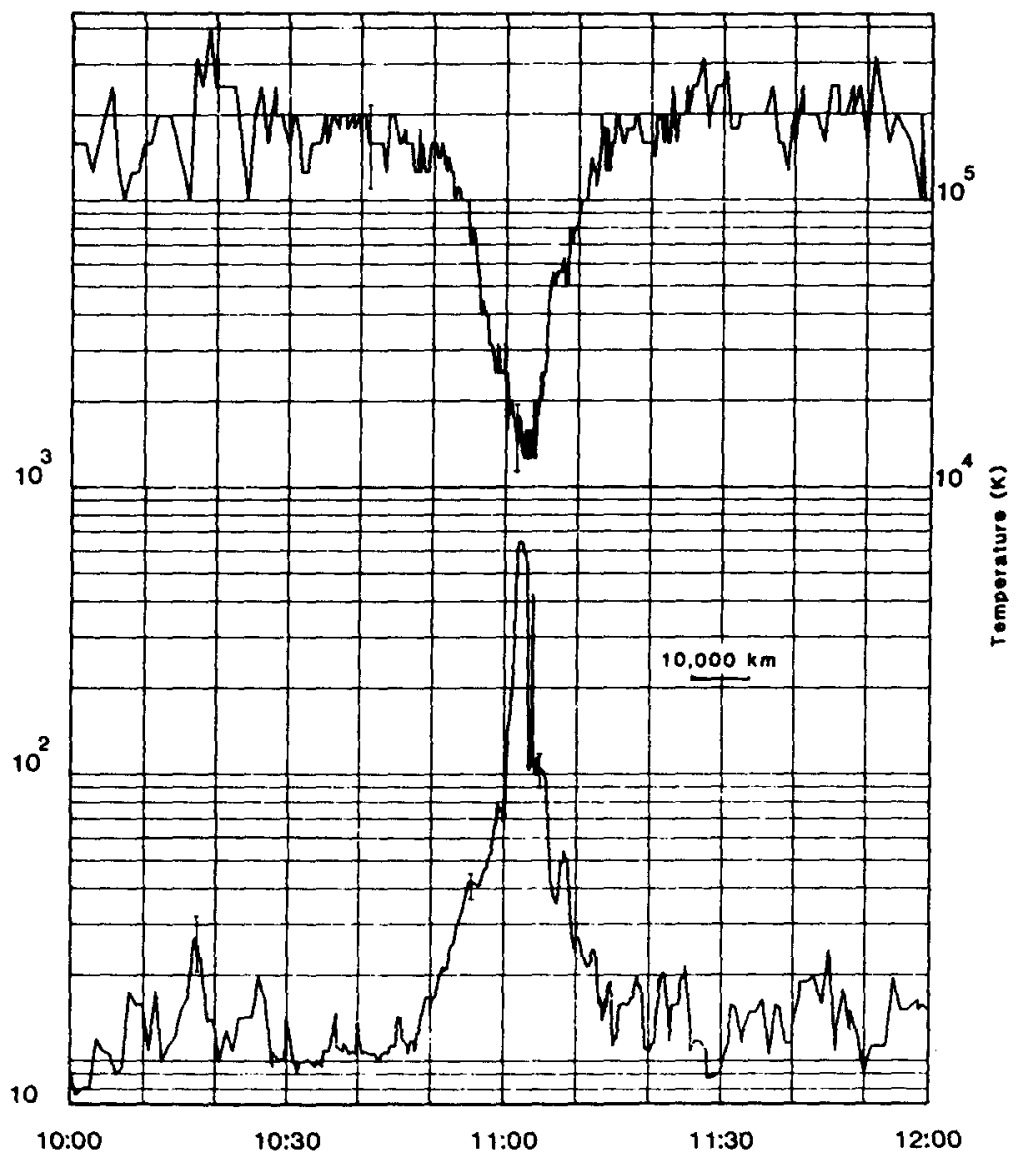

Fig. 6. Electron density and temperature time histories near the magnetotail of comet Giacobini-Zinner from the ICE radio experiment $/ 15 /$.

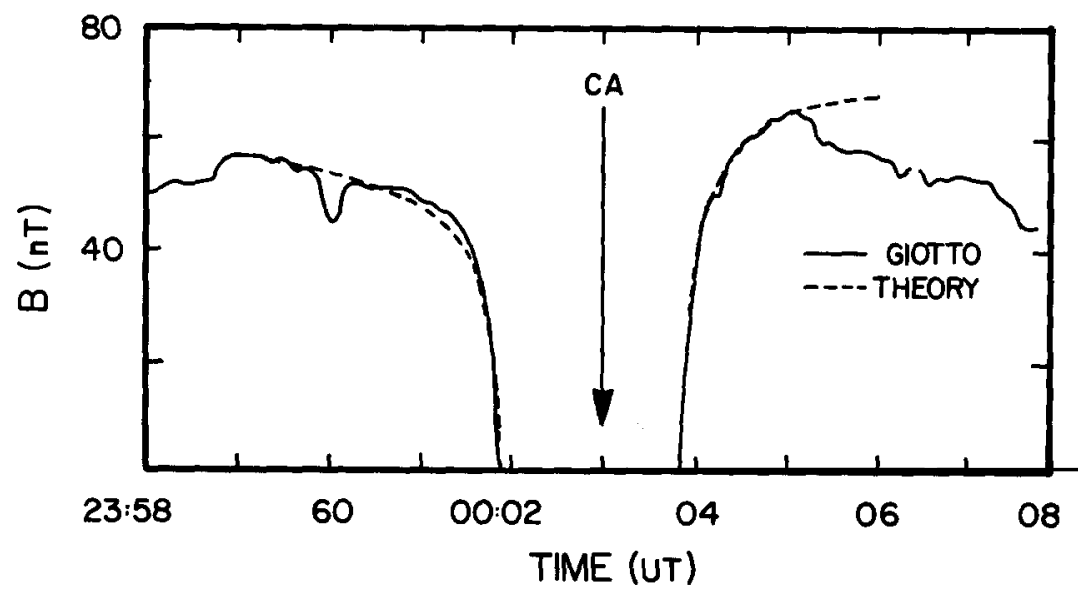

Fig. 7. Measured magnetic field magnitude versus time from the GIOTTO encounter with comet Halley /16/. CA designates the time of closest approach to the nucleus. A theoretically calculated magnetic field profile is also shown (see text). 


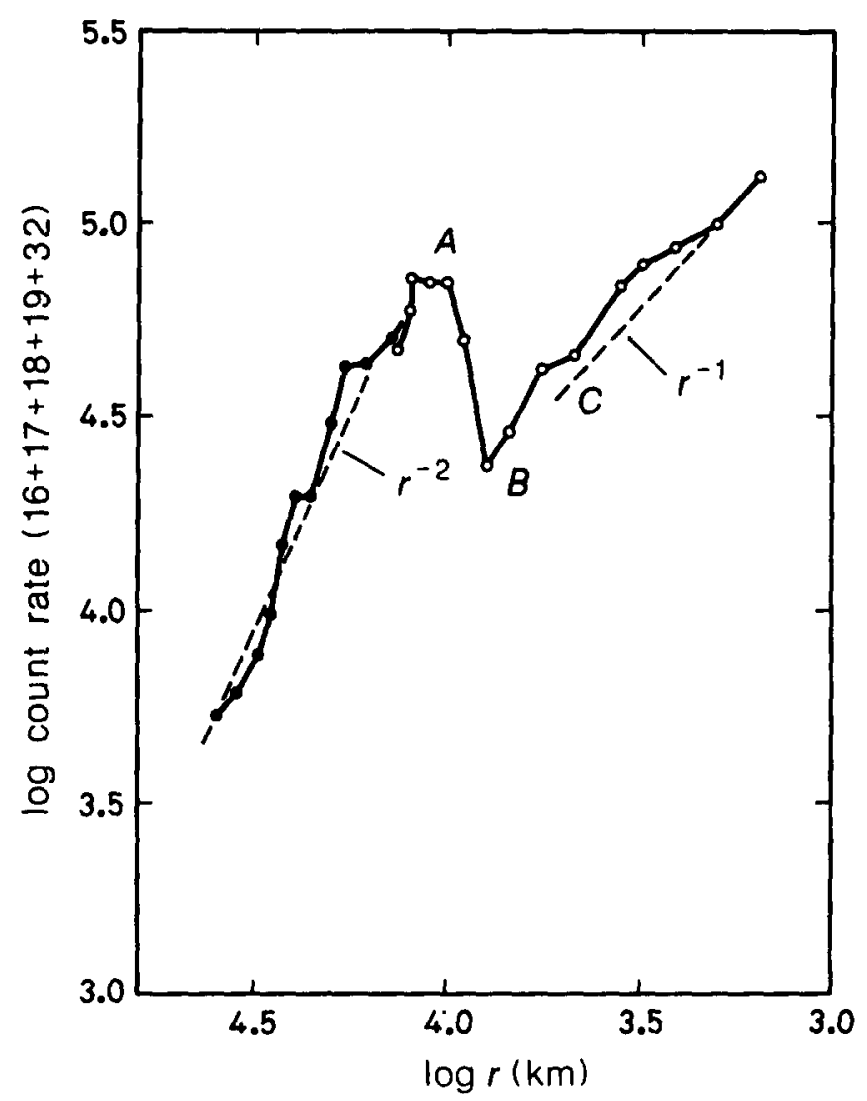

Fig. 8. Radial profile of counting rate for sum of masses $16,17,18,19$ and 32 from the HIS ion mass spectrometer on GIOTTO $/ 4 /$. C designates the contact surface.

All the criteria for ionospheric plasma are met and the plasma in the region just outside the contact surface should also be considered ionospheric. Hence, there are two ionospheres, or two parts to the ionosphere -- an inner field-free ionosphere (the "classic" ionosphere which the theoretical models have considered) and an outer magnetized ionosphere. Most of the above properties of the magnetized "ionosphere" were recognized to some extent $/ 1,2,11 /$ prior to the recent spacecraft encounters with comets Halley and $G / Z$, but the ionospheric nature of this region has not yet been fully recognized.

The plasma analyzers on the VEGA spacecraft observed a "cometary plasma region" in which the ions are very slow-moving and are entirely of cometary origin $/ 5 \%$. The outer boundary of this region has been called the cometopause and is located near $10^{5} \mathrm{~km}$ from the nucleus. The magnetized ionosphere discussed here is located in the inner part of the cometary plasma region.

\section{THE CONTACT SURFACE}

The contact surface can be defined as that surface where the magnetic field becomes zero, or extremely small $/ 16 /$ One can also define the contact "surface" as that region where the field goes from its external (or magnetic barrier) value to its very small ionospheric value, and in this case the CS is a region with finite thickness $(\approx$ few hundred-km, $/ 16 /$. The available data clearly demonstrate that the plasma pressure internal to the CS cannot balance the sum of the external plasma pressure and the external magnetic pressure. In fact, the plasma density on both sides of the CS is about the same; there is no Venus-like ionopause. The external magnetic pressure in the flanks of the comet Halley magnetic barrier (or stagnation region $/ 19 /$ or cometary plasma region $/ 5 /$ or inner plasma mantle $16 /$ ) is about $1.5 \times 10^{-8} \mathrm{dynes} / \mathrm{cm}^{2}$, whereas the internal plasma pressure and/or the internal dynamic pressure are both less than $5 \times 10^{-10}$ dynes $/ \mathrm{cm}^{2}$ and therefore cannot play an important role in the momentum balance near the CS. 


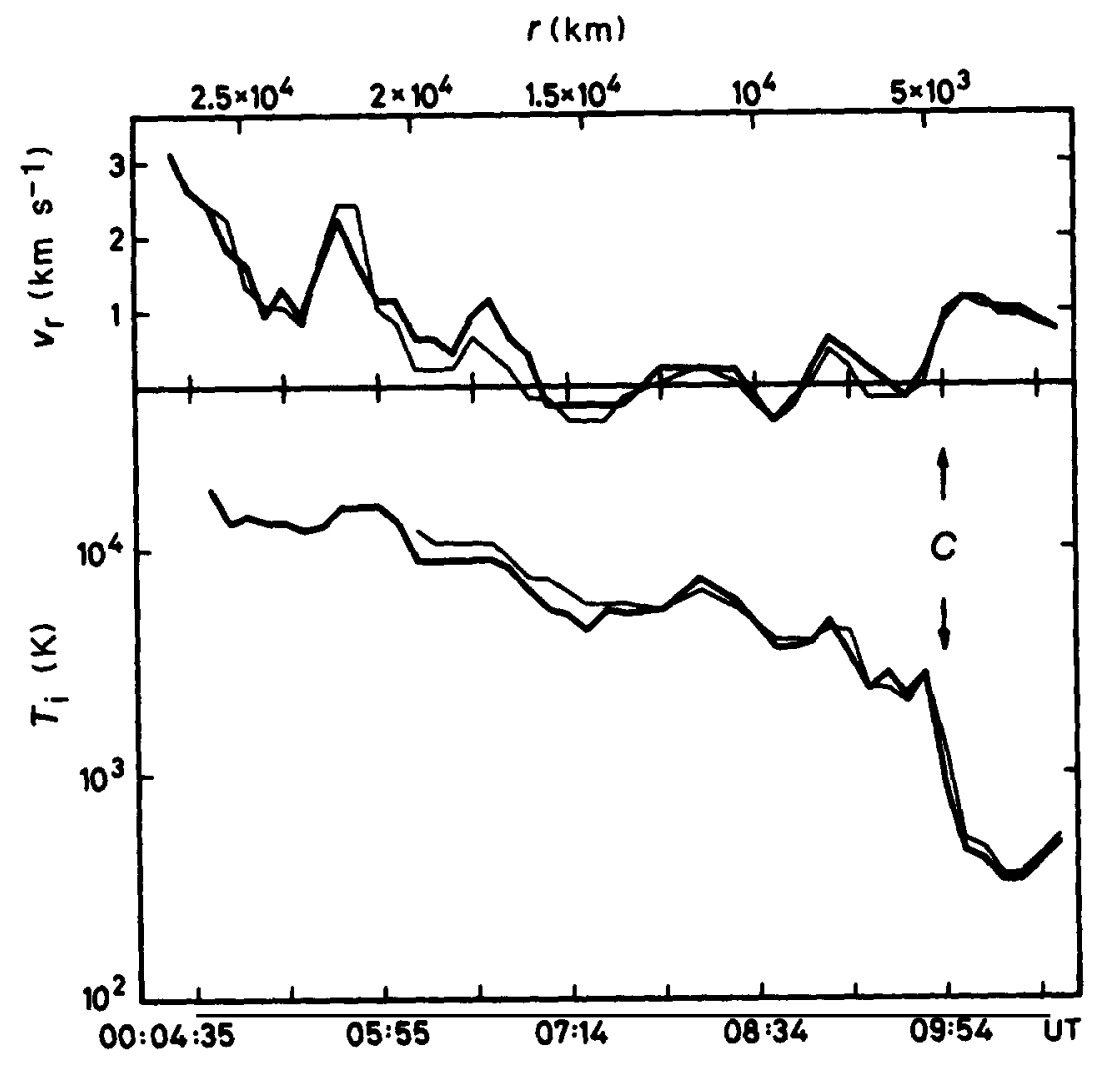

Fig. 9. Time history of both ion flow speed and temperature for $\mathrm{H}_{2} \mathrm{O}^{+}$(bold line) and $\mathrm{H}_{3} \mathrm{O}^{+}$(light line) from the GIOTTO IMS (from /4/).

The most important terms in the momentum equation for the conditions found near the CS are the $\mathbf{J} \times \mathbf{B}$ term (where $\mathbf{J}$ is the current density) and the ion-neutral drag term. The magnetic pressure gradient force is much larger than magnetic tension near the contact surface because the width of the CS $(\Delta r \approx 500-\mathrm{km})$ is much less than the radius of curvature which is greater than or equal to the radial distance to the contact surface ( $r_{c s} \cong 4500 \mathrm{~km}$ where $r_{\mathrm{cs}}$ is the cometocentric distance of the CS). Hence, using Amperre's law and neglecting magnetic tension it can be shown in one-dimension that $\mathbf{J} \times \mathbf{B} \cong-\partial / \partial \mathrm{r}\left[\mathrm{B}^{2} / 8 \pi\right]$, assuming that the radius vector is approximately normal to the contact surface in the flanks where GIOTTO crossed the CS. The momentum balance can then be written as:

$$
-\partial / \partial r\left[B^{2} / 8 \pi\right]=n_{e} m_{i} v_{i n}\left(u_{i}-u_{n}\right)
$$

where $m_{i}$ is the ion mass and $v_{\text {in }}$ is the ion-neutral momentum transfer collision frequency. One can write $v_{\text {in }}=k_{\text {in }}$ $\mathrm{n}_{\mathrm{n}}$ where $\mathrm{k}_{\mathrm{in}} \cong 2 \times 10^{-9} \mathrm{~cm}^{3} \mathrm{~s}^{-1}$. This momentum balance is schematically represented in Figure 10. Equation (9) can be integrated if a couple of assumptions/approximations are made. First, make the reasonable approximation that the electron density varies as $n_{e}=\left(n_{e o} r_{e o}\right) / r$ where $n_{e o}{ }_{e o}$ is given by equation (7b) for comet Halley. Second, assume that the ion velocity is zero in the vicinity of the CS $\left(u_{i}=0\right)$. The GIOTTO IMS data indicates that both these assumptions are reasonably good. Equation (9) then becomes:

$$
-\partial / \partial \mathrm{r}\left[\mathrm{B}^{2} / 8 \pi\right]=-\mathrm{YQ} / \mathrm{r}^{3}
$$

where the constant $Y=n_{e o} r_{e o} m_{i} k_{i n} / 4 \pi$. 


\section{INSIDE CONTACT SURFACE}

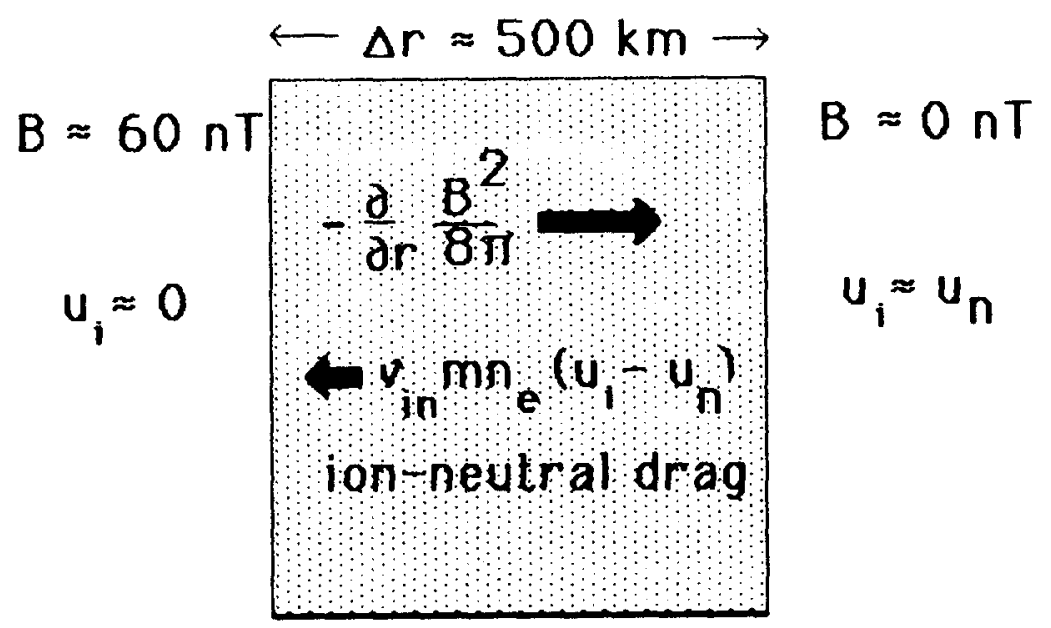
Fig. 10. Schematic of momentum balance across the contact surface which is shown as a "layer"
of finite thickness.

One finds that $\mathrm{Y}=6.8 \times 10^{-21}$ for comet Halley using values for the relevant parameters given earlier throughout this paper. One can assume that $T_{e} \approx 10^{3} \mathrm{~K}$. Equation (10) can be integrated from $\mathrm{r}=\infty$, where the magnetic field strength is equal to that in the magnetic barrier $\left(B=B_{o}\right)$, down to any value of $r$ greater than $r_{c s}$ :

$$
B(r)=B_{0}\left[1-\left(r_{c s} / r\right)^{2}\right]^{1 / 2}
$$

The distance to the contact surface is defined as that value of $r$ where $B(r)$ becomes zero:

$$
\mathrm{r}_{\mathrm{cS}}=[8 \pi \mathrm{YQ}]^{1 / 2} / \mathrm{B}_{\mathrm{o}}
$$

Note that the distance to the CS decreases as the field in the magnetic barrier, $\mathrm{B}_{\mathrm{o}}$, increases. The magnetic pressure $\left(\mathrm{B}^{2} / 8 \pi\right)$ in the magnetic barrier is approximately equal to the solar wind dynamic pressure (see references listed in $/ 99 \%$, and therefore as the solar wind dynamic pressure increases, the distance to the contact surface decreases (as one would expect). Equation (12) predicts that the distance to the contact surface increases as the gas production rate $Q$ increases. Actually, the dependence on gas production rate is $Q^{3 / 4}$, rather than the $Q^{1 / 2}$ dependence indicated formally in equation (12), because the factor $\mathrm{n}_{\mathrm{eo}} \mathrm{r}_{\mathrm{eg}}$ varies approximately as the one-half power of $\mathrm{Q}$ according to equation (7a). One can predict that the distance to the contact surface for comet Giacobini-Zinner is about $500 \mathrm{~km}$ for typical solar wind conditions by scaling from the $r_{c s} \approx 4500 \mathrm{~km}$ measured for comet Halley and using the fact that the gas production rate at $\approx 1 \mathrm{AU}$ of comet $\mathrm{G} / \mathrm{Z}$ is about a factor of 20 smaller than Halley's. The width of the contact surface, $\Delta r_{c s}$, can be defined as the extent of the region between $r_{c s}$ and where $B=0.5 B_{0}$, in which case from equation (11) one finds that $\Delta \mathrm{r}_{\mathrm{cs}} \cong 0.15 \mathrm{r}_{\mathrm{cs}}(\approx 700 \mathrm{~km}$ for Halley).

Equation (11) was used to model the variation of the magnetic field with $\mathrm{r}$ at the time of the GIOTTO encounter. The inbound and outbound data was modeled separately. In each case, the value of $r_{c s}$ was chosen to match the observed value ( $4760 \mathrm{~km}$ inbound and $3840 \mathrm{~km}$ outbound) and $B_{0}$ was then chosen to find the best agreement between equation (11) and the magnetic field data. This procedure gives $B_{0}=57 \mathrm{nT}$ inbound and $70.7 \mathrm{nT}$ outbound. The comparison of theory and measurements is shown in Figure 7, and the agreement is very good. Equation (12) can then be used to find values of $Q$ for both the inbound and outbound legs of the GIOTTO trajectory since $r_{c s}$ and $B_{o}$ were chosen to "fit" the observed field. This procedure gives $Q=4.3 \times 10^{29} \mathrm{~s}^{-1}$ for both inbound and outbound (to within less than $1 \%$ ). This value of $Q$ is about $50 \%$ smaller than the gas production rate measured by the GIOTTO NMS /3/; however, the agreement is excellent considering the various uncertainties in the constant $Y$ and the neglected terms in the momentum equation. 
Now reconsider the very important assumption that $u_{i}=0$. The IMS data [4] supports this assumption near the contact surface but there are also theoretical reasons for expecting very small ion velocities near the CS. A very small $\mathrm{u}_{\mathrm{i}}$ follows from basic MHD considerations and is a consequence of two factors: (1) steady-state conditions and (2) frozen-in magnetic flux. The standard equation for B, assuming that the Ohmic resistivity is very small, is

$$
\partial \mathbf{B} / \partial \mathrm{t}=\nabla \times\left(\mathbf{u}_{\mathbf{i}} \times \mathbf{B}\right)
$$

Equation (13) does not include a magnetic "diffusion" term, which it should if the Ohmic resistivity were significant (cf. /20/; however, the diffusion term is roughly $10^{3}$ times smaller than the magnetic covection term (which was included in equation (13)) at distances of several thousand $\mathrm{km}$.

For one-dimensional conditions (that is, neglecting the non-radial convection of magnetic flux which probably does play some role), equation (13) becomes $\partial \mathrm{B} / \partial \mathrm{t}=-\partial\left[\mathrm{u}_{\mathrm{i}} \mathrm{B}\right] / \partial \mathrm{r}$. The steady-state solution of this equation is $\mathrm{u}_{\mathrm{i}} \mathrm{B}$ $=$ constant. The magnetic field within the ionosphere is almost zero and the velocity is about equal to the neutral velocity $u_{n}$. This suggests that the ion velocity decreases rapidly as $B$ increases, so that within the contact surface $u_{i}$ is very small where $B$ is large.

Now consider how the magnetic field (and contact surface) responds to changing solar wind dynamic pressure. When the solar wind dynamic pressure increases, the magnetic field in the barrier / stagnation region (i.e., $\mathrm{B}_{\mathrm{o}}$ ) will increase, and from equation (12) the distance to the contact surface will decrease. How does this happen? Equation (9) indicates that, initially, $u_{i}$ becomes increasingly negative (inward) in response to an increased pressure gradient force which is unbalanced by the outward ion-neutral drag. Magnetic flux is pushed inward as a consequence of the now inwardly flowing plasma (a consequence of equation (13)). The region of the maximum gradient of $B$ continues to push inward until the ion-neutral drag term (which varies as $1 / r^{3}$ ) has built up sufficiently so as to again balance the pressure gradient force. The ion velocity then decreases back to zero again as a new equilibrium momentum balance is set up. The new equilibrium position of the CS is now closer to the nucleus than when this process started. Conversely, if the solar wind dynamic pressure decreases, ion-neutral drag produces outward flow which convects magnetic flux (and the CS) outward.

Earlier theoretical discussions recognized the importance of ion-neutral drag in the vicinity of the contact surface / cf., $1,2,19,21 /$, but an ionopause was expected to form by analogy with Venus. It was expected /cf., 1,2,19,21/ that ion-neutral drag would enhance the thermal plasma pressure between the inner shock and the CS and thus increase the ionopause distance. However, the IMS measurements /4/clearly indicate the electron density barely deviates from $1 / \mathrm{r}$ throughout the $\mathrm{CS}$ region. In retrospect, this makes sense if one considers that the chemical lifetime (i.e., the recombination time) of the ions is comparable to the ion transport time even if $u_{i} \approx u_{n}$ and, therefore, must be much shorter than the transport time near the contact surface where $u_{i} \approx 0$. The ions are photochemically controlled in this region which means that no matter how "one pushes them around" the density is going to be the photochemically controlled and the ions will not "feel" the existence of the CS.

\section{SUMMARY}

The basic physical and chemical processes responsible for the ionospheres of comets were reviewed in this paper. Both the current theoretical picture and the current experimental picture of cometary ionospheres was presented. It was suggested that the plasma just outside the contact surface is just as ionospheric in nature as the "classical" ionospheric plasma residing within this surface. Finally, the processes responsible for the formation of the contact surface were discussed and an expression was derived for the magnetic field in this region.

\section{ACKNOWLEDGMENTS}

This work was supported by NASA grants NAGW-15, NGR 23-005-015 and NSF grant ATM 8417884. The author thanks J.H. Luhmann, T.I. Gombosi, and A.F. Nagy for useful discussions.

\section{REFERENCES}

1. D.A. Mendis, H.L.F. Houpis and M.L. Marconi, The physics of comets, Fund, Cosmic Phys., 10, 1, (1985)

2. W.H. Ip and W.I. Axford, Theories of physical processes in the cometary comae and ion tails, Comets, ed. L.L. Wilkening, Tucson, Arizona, University of Arizona Press, 588, 1982.

3. D. Krankowsky, P. Lämmerzahl, I. Herrwerth, J. Woweries, P. Eberhardt, U. Dolder, U. Hermann, W. Schulte, J.J. Berthelier, J.M. Illiano, R.R. Hodges and J.H. Hoffman, In situ gas and ion measurements at comet Halley, Nature, 321, 326 (1986) 
4. H.K. Balsiger, K. Altwegg, J. Geiss, A.G. Ehielmetti, B.E. Goldstein, R. Goldstein, W.T. Huntress, W-H Ip, A.J. Lazarus, A. Meier, M. Neugebauer, U. Rettenmudd, H. Rosenbauer, R. Schwenn, R.D. Sharp, E.G. Shelley, E. Ungstrup and D.T. Young, Ion comparison and dynamics at comet Halley, Nature, 321, 330 (1986)

5. K.I. Gringauz, T.I. Gombosi, A.P. Remizov, I. Apáthy, I. Szemerey, M.I. Verigin, L.I. Denchikova, A.V. Dyachkov, E. Keppler, I.N. Klimenko, A.K. Richter, A.J. Somogyi, K. Szego, S. Szendro, M Tátrallyay, A. Varga and G.A. Vladimirova, First in situ plasma and neutral gas measurements at comet Halley, Nature, $321,282(1986)$

6. T.E. Cravens, J.U. Kozyra, A.F. Nagy, T.I. Gombosi and M. Kurtz, Electron Impact ionization in the vicinity of comets, to be submitted to $\mathrm{J}$, Geophys. Res., (1986)

7. M.L. Marconi, D.A. Mendis, The electron density and temperature in the tail of comet Giacobini-Zinner, Geophys. Res. Lett., 13405 (1986)

8. A. Körösmezey, T.E. Cravens, T.I. Gombosi, A.F. Nagy, D.A. Mendis, K. Szego, B.E. Gribov, R.Z. Sageev, V. D. Shapiro and V.I. Shevchenko, A comprehensive model of cometary ionospheres, Plamet. Space Sci: in press (1986)

9. T.I. Gombosi, M. Horanyi, K. Kecskemety, T.E. Cravens and A.F. Nagy, Charge exchange in solar wind cometary interactions, Astrophys, J. 268, 889 (1983)

10. W.F. Huebner, P.T. Giguere, and W.L. Slattery, Photochemical processes in comets, Comets, ed. L.L. Wilkening , Univ. of Arizona Press, Tucson 1982, p. 496.

11. M.L. Marconi and D.A. Mendis, The atmosphere of a dirth clathrate cometary nucleus: A tow-phase multi-fluid model, Astrophys. J. 381 (1983)

12 M.L. Marconi and D.A. Mendis, The effects of diffuse radiation fields due to multiple scattering and thermal re-radiation by dust on the dynamics and thermodynamics of a dusty cometary atmosphere, Astrophys. $J$. 287, 445 (1984)

13. T.I. Gombosi, A.F. Nagy and T.E. Cravens, Dust and neutral gas modeling of the inner atmospheres of comets, Rev. Geophys, in press (1986)

14. J.A. Slavin, E.J. Smith, B.T. Tsurutani, G.L. Siscoe, D.E. Jones, and D.A. Mendis, Giacobini-Zinner magnetotail: ICE magnetic field observations, Geophys. Res. Lett. 13, 283 (1986).

15. N. Meyer-Vernet, P. Couturier, S. Hoang, C. Perche, J.L. Steinberg, J. Fainberg, C. Meetre, Plasma diagnosis from thermal noise and limits on dust flux or mass in comet P/Giacobini-Zinner, Science, 232 (1986)

16. F.M. Neubauer, K.H. Glassmeier, M. Pohl, J. Raeder, M.H. Acuna, L.F. Burlaga, N.F. Ness, G. Mussman, F. Mariani, M.V. Wallis, E. Ungstrop and H.U. Schmidt, First results from the Giotto magnetometer experiment at comet Halley, Nature, 321, 352 (1986)

17. M.K. Wallis and R.S.B. Ong, Cooling and recombination processes in cometary plasmas, in: the Study of Comets, eds. B. Donn, M. Mumma, W. Jackson, M. A'Hearn, and R. Harrington, NASA SP-393, Washington 1976, p. 856

18. T.E. Cravens, A. Körösmezey, Vibrational and cooling of electrons by water vapor, Planetary Space Science, in press (1986)

19. A.A. Galeev, T.E. Cravens, and T.I. Gombosi, Solar wind stagnation near comets, Astrophys. J., 289,807 (1985)

20. T.E. Cravens, H. Shinagawa and A.F. Nagy, The evolution of large-scale magnetic fields in the ionosphere of Venus, Geophys. Res. Lett., 11267 (1984)

21. D.A. Mendis and H.L.F. Houpis, The cometary atmosphere and its interaction with the solar wind, $\underline{\operatorname{Rev}}$ Geophys. Res., 20, 885 (1982) 